FACTA UNIVERSITATIS (NIŠ)

Ser. Math. Inform. Vol. 34, No 5 (2019), 855-869

https://doi.org/10.22190/FUMI1905855K

\title{
EVALUATION OF A NEW CLASS OF EULERIAN'S TYPE INTEGRALS INVOLVING GENERALIZED HYPERGEOMETRIC FUNCTIONS*
}

\author{
Insuk Kim, Gradimir V. Milovanović, and Arjun K. Rathie
}

(C) 2019 by University of Niš, Serbia | Creative Commons Licence: CC BY-NC-ND

Abstract. Very recently Masjed-Jamei and Koepf [Axioms 2018, 7 (2), 38] established interesting and useful generalizations of various classical summation theorems for the ${ }_{2} F_{1},{ }_{3} F_{2},{ }_{4} F_{3},{ }_{5} F_{4}$ and ${ }_{6} F_{5}$ generalized hypergeometric series. The main aim of this paper is to establish eleven Eulerian's type integrals involving generalized hypergeometric functions by employing these theorems. Several special cases (known and unknown) have also been given.

Keywords: Generalized hypergeometric functions, classical summation theorems generalization

\section{Introduction}

We define the well known Pochhammer symbol, for any complex number $a$ as

$$
(a)_{n}=\frac{\Gamma(a+n)}{\Gamma(a)}= \begin{cases}1 & (n=0, a \in \mathbb{C} \backslash\{0\}), \\ a(a+1) \cdots(a+n-1) & (n \in \mathbb{N}, a \in \mathbb{C}),\end{cases}
$$

where $\Gamma(z)$ is the well known gamma function defined by

$$
\Gamma(z)=\int_{0}^{\infty} \mathrm{e}^{-x} x^{z-1} \mathrm{~d} x
$$

for $\operatorname{Re}(z)>0$.

Received August 10, 2019; accepted November 24, 2019

2010 Mathematics Subject Classification. Primary 33C20; Secondary 33C05, 65B10.

* This work was supported by Wonkwang University in 2018. The second author was supported in part by the Serbian Academy of Sciences and Arts (No. $\Phi-96$ ). 
Based on Pochhammer symbol (1.1), the generalized hypergeometric function with $p$ numerator and $q$ denominator parameters is defined $[1,2,8,9]$ as

$$
{ }_{p} F_{q}\left[\begin{array}{ccc}
a_{1}, & \ldots, & a_{p} \\
b_{1}, & \ldots, & b_{q}
\end{array} ;\right]=\sum_{n=0}^{\infty} \frac{\left(a_{1}\right)_{n} \cdots\left(a_{p}\right)_{n}}{\left(b_{1}\right)_{n} \cdots\left(b_{q}\right)_{n}} \cdot \frac{z^{n}}{n !} .
$$

It can be easily verified by the ratio test (cf. [1]) that the series (1.2) is convergent for all $p \leq q$. Also it converges in $|z|<1$ for $p=q+1$ and converges everywhere for $p<q+1$ and converges nowhere $(z \neq 0)$ for $p>q+1$.

Further, if $p=q+1$, it converges absolutely for $|z|=1$ provided

$$
\delta=\operatorname{Re}\left(\sum_{j=1}^{q} b_{j}-\sum_{j=1}^{p} b_{j}\right)>0
$$

holds and is conditionally convergent for $|z|=1$ and $z \neq 1$ if $-1<\delta \leq 0$ and diverges for $|z|=1$ and $z \neq 1$ if $\delta \leq-1$ (for more details, we refer [8]).

The two very important cases of the series (1.2), viz.

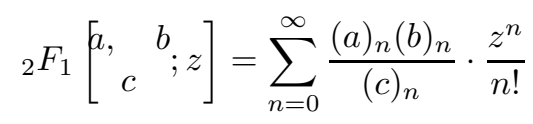

converges for $|z| \leq 1$ and

$$
{ }_{1} F_{1}\left[\begin{array}{l}
a \\
c
\end{array} z\right]=\sum_{n=0}^{\infty} \frac{(a)_{n}}{(c)_{n}} \cdot \frac{z^{n}}{n !}
$$

converges everywhere, popularly known in the literature on the Gauss's hypergeometric function and Kummer's function or confluent hypergeometric function arising in many physical problems $[5,6]$.

It is evident that whenever a generalized hypergeometric functions reduce to gamma function, the result are very important from the application point of view.

Moreover, here we mention the following classical summation theorems recorded in $[1,2]$ :

- Gauss Theorem

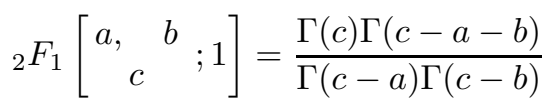

provided $\operatorname{Re}(c-a-b)>0$;

- Kummer's Theorem

$$
{ }_{2} F_{1}\left[\begin{array}{c}
a, b \\
1+a-b
\end{array} ;-1\right]=\frac{\Gamma(1+a-b) \Gamma\left(1+\frac{1}{2} a\right)}{\Gamma\left(1-b+\frac{1}{2} a\right) \Gamma(1+a)}
$$


- Second Gauss Theorem

$$
{ }_{2} F_{1}\left[\begin{array}{c}
a, \quad b \\
\frac{1}{2}(a+b+1)
\end{array} ; \frac{1}{2}\right]=\frac{\sqrt{\pi} \Gamma\left(\frac{1}{2}(a+b+1)\right)}{\Gamma\left(\frac{1}{2}(a+1)\right) \Gamma\left(\frac{1}{2}(b+1)\right)} ;
$$

- Bailey's Theorem

$$
{ }_{2} F_{1}\left[\begin{array}{lll}
a, & 1-a \\
& b & ;
\end{array}\right]=\frac{\Gamma\left(\frac{1}{2} b\right) \Gamma\left(\frac{1}{2}(b+1)\right)}{\Gamma\left(\frac{1}{2}(a+b)\right) \Gamma\left(\frac{1}{2}(b-a+1)\right)} ;
$$

- Dixon's Theorem

$$
\begin{aligned}
& { }_{3} F_{2}\left[\begin{array}{c}
a, \quad b, \quad c \\
1+a-b, \quad 1+a-c
\end{array} ; 1\right] \\
& =\frac{\Gamma\left(1+\frac{1}{2} a\right) \Gamma(1+a-b) \Gamma(1+a-c) \Gamma\left(1-b-c+\frac{1}{2} a\right)}{\Gamma(1+a) \Gamma\left(1-b+\frac{1}{2} a\right) \Gamma\left(1-c+\frac{1}{2} a\right) \Gamma(1+a-b-c)}
\end{aligned}
$$

provided $\operatorname{Re}(a-2 b-2 c)>-2$;

- Watson's Theorem

$$
\begin{array}{r}
{ }_{3} F_{2}\left[\begin{array}{ccc}
a, & b, & c \\
\frac{1}{2}(a+b+1), & 2 c & ; 1
\end{array}\right] \\
=\frac{\sqrt{\pi} \Gamma\left(c+\frac{1}{2}\right) \Gamma\left(\frac{1}{2}(a+b+1)\right) \Gamma\left(c-\frac{1}{2}(a+b-1)\right)}{\Gamma\left(\frac{1}{2}(a+1)\right) \Gamma\left(\frac{1}{2}(b+1)\right) \Gamma\left(c-\frac{1}{2}(a-1)\right) \Gamma\left(c-\frac{1}{2}(b-1)\right)}
\end{array}
$$

provided $\operatorname{Re}(2 c-a-b)>-1$;

- Whipple's Theorem

$$
\begin{aligned}
& { }_{3} F_{2}\left[\begin{array}{cc}
a, & 1-a, b \\
c, & 2 b-c+1
\end{array} ; 1\right] \\
& =\frac{\pi 2^{1-2 b} \Gamma(c) \Gamma(2 b-c+1)}{\Gamma\left(\frac{1}{2}(a+c)\right) \Gamma\left(b+\frac{1}{2}(a-c+1)\right) \Gamma\left(\frac{1}{2}(1-a+c)\right) \Gamma\left(b+1-\frac{1}{2}(a+c)\right)} ;
\end{aligned}
$$

- Pfaff-Saalschütz Theorem

$$
{ }_{3} F_{2}\left[\begin{array}{c}
a, \quad b, \quad-n \\
c, \quad 1+a+b-c-n
\end{array} ; 1\right]=\frac{(c-a)_{n}(c-b)_{n}}{(c)_{n}(c-a-b)_{n}}
$$


- Second Whipple's Theorem

$$
{ }_{4} F_{3}\left[\begin{array}{c}
a, \quad 1+\frac{1}{2} a, \quad b, \quad c \\
\frac{1}{2} a, a-b+1, a-c+1
\end{array} ;-1\right]=\frac{\Gamma(a-b+1) \Gamma(a-c+1)}{\Gamma(a+1) \Gamma(a-b-c+1)} ;
$$

- Dougall's Theorem

$$
\begin{array}{r}
{ }_{5} F_{4}\left[\begin{array}{c}
a, \quad 1+\frac{1}{2} a, \quad c, \quad d, \quad e \\
\frac{1}{2} a, \quad a-c+1, \quad a-d+1, \quad a-e+1
\end{array}\right] \\
=\frac{\Gamma(a-c+1) \Gamma(a-d+1) \Gamma(a-e+1) \Gamma(a-c-d-e+1)}{\Gamma(a+1) \Gamma(a-d-e+1) \Gamma(a-c-e+1) \Gamma(a-c-d+1)}
\end{array}
$$

- Second Dougall's Theorem

$$
\begin{gathered}
{ }_{7} F_{6}\left[\begin{array}{c}
a, \quad 1+\frac{1}{2} a, \quad b, \quad c, \quad d, \quad 1+2 a-b-c-d+n, \quad-n \\
\frac{1}{2} a, a-b+1, a-c+1, a-d+1, b+c+d-a-n, a+1+n
\end{array} ; 1\right] \\
=\frac{(a+1)_{n}(a-b-c+1)_{n}(a-b-d+1)_{n}(a-c-d+1)_{n}}{(a+1-b)_{n}(a+1-c)_{n}(a+1-d)_{n}(a+1-b-c-d)_{n}}
\end{gathered}
$$

For finite sums of hypergeometric series, we will use the following symbol

$$
{ }_{p}^{(m)} F_{q}\left[\begin{array}{lll}
a_{1}, & \ldots, & a_{p} \\
b_{1}, & \ldots, & b_{q}
\end{array} ; z=\sum_{n=0}^{m} \frac{\left(a_{1}\right)_{n} \cdots\left(a_{p}\right)_{n}}{\left(b_{1}\right)_{n} \cdots\left(b_{q}\right)_{n}} \cdot \frac{z^{n}}{n !}\right.
$$

where for instance

$$
{ }_{p}^{(-1)} F_{q}(z)=0, \quad \stackrel{(0)}{F_{q}}(z)=1, \quad{ }_{p} F_{q}(z)=1+\frac{a_{1} \cdots a_{p}}{b_{1} \cdots b_{q}} z .
$$

By using the following relation [7],

$$
\begin{aligned}
{ }_{p} F_{q}\left[\begin{array}{l}
a_{1}, \ldots, a_{p-1}, 1 \\
b_{1}, \ldots, b_{q-1}, m
\end{array} ; z\right] \\
=\frac{\Gamma\left(b_{1}\right) \cdots \Gamma\left(b_{q-1}\right)}{\Gamma\left(a_{1}\right) \cdots \Gamma\left(a_{p-1}\right)} \frac{\Gamma\left(a_{1}-m+1\right) \cdots \Gamma\left(a_{p-1}-m+1\right)}{\Gamma\left(b_{1}-m+1\right) \cdots \Gamma\left(b_{q-1}-m+1\right)} \frac{(m-1) !}{z^{m-1}} \\
\quad \times\left\{{ }_{p-1} F_{q-1}\left[\begin{array}{c}
a_{1}-m+1, \ldots, a_{p-1}-m+1 \\
b_{1}-m+1, \ldots, b_{q-1}-m+1
\end{array} ;\right]\right. \\
(m-2) \\
\left.-{ }_{p-1} F_{q-1}\left[\begin{array}{c}
a_{1}-m+1, \ldots, a_{p-1}-m+1 \\
b_{1}-m+1, \ldots, b_{q-1}-m+1
\end{array} ; z\right]\right\},
\end{aligned}
$$

very recently Masjed-Jamei and Koepf [4] have established generalizations of the classical summation formulas (1.3)-(1.13) in the following forms: 
(1.14)

$$
\begin{aligned}
& { }_{3} F_{2}\left[\begin{array}{c}
a, b, 1 \\
c, m
\end{array} ; 1\right]=\frac{\Gamma(m) \Gamma(c) \Gamma(a-m+1) \Gamma(b-m+1)}{\Gamma(a) \Gamma(b) \Gamma(c-m+1)} \\
& \quad \times\left\{\frac{\Gamma(c-m+1) \Gamma(c-a-b+m-1)}{\Gamma(c-a) \Gamma(c-b)}-{ }_{2}^{(m-2)} F_{1}\left[\begin{array}{c}
a-m+1, b-m+1 \\
c-m+1
\end{array} ; 1\right]\right\}=\Omega_{1} ;
\end{aligned}
$$

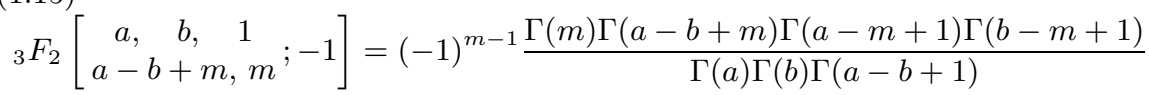

$$
\begin{aligned}
& \times\left\{\frac{\Gamma(a-b+1) \Gamma\left(1+\frac{1}{2}(a-m+1)\right)}{\Gamma(2+a-m) \Gamma\left(m-b+\frac{1}{2}(a-m+1)\right)}-{ }_{2}^{(m-2)} F_{1}\left[\begin{array}{c}
a-m+1, b-m+1 \\
a-b+1
\end{array} ;-1\right]\right\}=\Omega_{2} ;
\end{aligned}
$$

$$
\begin{aligned}
& { }_{3} F_{2}\left[\begin{array}{c}
a, \quad b, \quad 1 \\
\frac{1}{2}(a+b+1), m
\end{array} ; \frac{1}{2}\right]=2^{m-1} \frac{\Gamma(m) \Gamma\left(\frac{1}{2}(a+b+1)\right) \Gamma(a-m+1) \Gamma(b-m+1)}{\Gamma(a) \Gamma(b) \Gamma\left(-m+1+\frac{1}{2}(a+b+1)\right)} \\
& \quad \times\left\{\frac{\sqrt{\pi} \Gamma\left(-m+1+\frac{1}{2}(a+b+1)\right)}{\Gamma\left(1+\frac{1}{2}(a-m)\right) \Gamma\left(1+\frac{1}{2}(b-m)\right)}-\stackrel{(m-2)}{{ }_{2} F_{1}}\left[\begin{array}{c}
a-m+1, b-m+1 \\
-m+1+\frac{1}{2}(a+b+1)
\end{array} ; \frac{1}{2}\right]\right\}=\Omega_{3} ;
\end{aligned}
$$

$$
\begin{aligned}
& { }_{3} F_{2}\left[\begin{array}{c}
a, 2 m-a-1,1 \\
b, \quad m
\end{array} ; \frac{1}{2}\right]=2^{m-1} \frac{\Gamma(m) \Gamma(b) \Gamma(a-m+1) \Gamma(m-a)}{\Gamma(a) \Gamma(2 m-a-1) \Gamma(b-m+1)} \\
& \times\left\{\frac{\Gamma\left(\frac{1}{2}(b-m+1)\right) \Gamma\left(\frac{1}{2}(b-m+2)\right)}{\Gamma\left(-m+1+\frac{1}{2}(a+b)\right) \Gamma\left(\frac{1}{2}(b-a+1)\right)}-{ }_{2}^{(m-2)} F_{1}\left[\begin{array}{c}
a-m+1, m-a \\
b-m+1
\end{array} ; \frac{1}{2}\right]\right\}=\Omega_{4} ;
\end{aligned}
$$

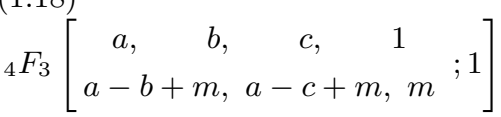

$$
\begin{aligned}
& =\frac{\Gamma(m) \Gamma(a-b+m) \Gamma(a-c+m) \Gamma(a+1-m) \Gamma(b+1-m) \Gamma(c+1-m)}{\Gamma(a) \Gamma(b) \Gamma(c) \Gamma(a-b+1) \Gamma(a-c+1)} \\
& \times\left\{\frac{\Gamma\left(\frac{1}{2}(a+3-m)\right) \Gamma(a-b+1) \Gamma(a-c+1) \Gamma\left(-b-c+\frac{1}{2}(a+3 m-1)\right)}{\Gamma(a+2-m) \Gamma\left(-b+\frac{1}{2}(a+m+1)\right) \Gamma\left(-c+\frac{1}{2}(a+m+1)\right) \Gamma(a-b-c+m)}\right. \\
& \left.-{ }_{3} F_{2}\left[\begin{array}{c}
a-m+1, b-m+1, c-m+1 \\
a-b+1, \quad a-c+1
\end{array}\right] 1\right\}=\Omega_{5} ;
\end{aligned}
$$


(1.19)

$$
\begin{array}{r}
\left.{ }_{4} F_{3}\left[\begin{array}{ccc}
a, \quad b, & c, & 1 \\
\frac{1}{2}(a+b+1), & 2 c+1-m, m
\end{array}\right] 1\right] \\
=\frac{\Gamma(m) \Gamma\left(\frac{1}{2}(a+b+1)\right) \Gamma(2 c+1-m) \Gamma(a+1-m) \Gamma(b+1-m) \Gamma(c+1-m)}{\Gamma(a) \Gamma(b) \Gamma(c) \Gamma\left(-m+\frac{1}{2}(a+b+3)\right) \Gamma(2 c-2 m+2)} \\
\quad \times\left\{\frac{\sqrt{\pi} \Gamma\left(c-m+\frac{3}{2}\right) \Gamma\left(-m+\frac{1}{2}(a+b+3)\right) \Gamma\left(c-\frac{1}{2}(a+b-1)\right)}{\Gamma\left(1+\frac{1}{2}(a-m)\right) \Gamma\left(1+\frac{1}{2}(b-m)\right) \Gamma\left(c+1-\frac{1}{2}(a+m)\right) \Gamma\left(c+1-\frac{1}{2}(b+m)\right)}\right. \\
\left.-{ }_{3}^{(m-2)}\left[\begin{array}{c}
a-m+1, b-m+1, c-m+1 \\
-m+1+\frac{1}{2}(a+b+1), 2 c-2 m+2
\end{array} ; 1\right]\right\}=\Omega_{6} ;
\end{array}
$$

$$
\begin{aligned}
& { }_{4} F_{3}\left[\begin{array}{cc}
a, 2 m-1-a, b, 1 & ; 1 \\
c, 2 b-c+1, m & ; 1
\end{array}\right] \\
& =\frac{\Gamma(m) \Gamma(c) \Gamma(2 b-c+1) \Gamma(m-a) \Gamma(a+1-m) \Gamma(b+1-m)}{\Gamma(a) \Gamma(b) \Gamma(2 m-1-a) \Gamma(c+1-m) \Gamma(2 b-c-m+2)} \\
& \times\left\{\frac{\pi 2^{2 m-2 b-1} \Gamma(c-m+1)}{\Gamma\left(-m+1+\frac{1}{2}(a+c)\right) \Gamma\left(-m+1+b+\frac{1}{2}(a-c+1)\right) \Gamma\left(\frac{1}{2}(1-a+c)\right)}\right. \\
& \left.\times \frac{\Gamma(2 b-c-m+2)}{\Gamma\left(b+1-\frac{1}{2}(a+c)\right)}-{ }_{3}^{(m-2)} F_{2}\left[\begin{array}{c}
a-m+1, b-m+1, m-a \\
c-m+1,2 b-c-m+2
\end{array} ; 1\right]\right\}=\Omega_{7} ;
\end{aligned}
$$

(1.21)

$$
\begin{aligned}
& { }_{4} F_{3}\left[\begin{array}{c}
a, b,-n+m-1,1 \\
c, 1+a+b-c-n, m
\end{array} ; 1\right]=\frac{(m-1) !(1-c)_{m-1}}{(1-a)_{m-1}(1-b)_{m-1}} \cdot \frac{(c-a-b+n)_{m-1}}{(n+2-m)_{m-1}} \\
& \times\left\{\frac{(c-a)_{n}(c-b)_{n}}{(c+1-m)_{n}(c-a-b+m-1)_{n}}-{ }_{3}^{(m-2)} F_{2}\left[\begin{array}{c}
a-m+1, b-m+1,-n \\
c-m+1,2+a+b-c-m-n
\end{array} ; 1\right]\right\}=\Omega_{8} ;
\end{aligned}
$$

$$
\begin{aligned}
& { }_{5} F_{4}\left[\begin{array}{c}
a, \quad \frac{1}{2}(a+m+1), \quad b, \quad c, \quad 1 \\
\frac{1}{2}(a+m-1), a-b+m, a-c+m, m
\end{array} ;-1\right] \\
& =(-1)^{m-1} \frac{\Gamma(m) \Gamma\left(\frac{1}{2}(a+m-1)\right) \Gamma(a-b+m) \Gamma(a-c+m)}{\Gamma(a) \Gamma(b) \Gamma(c) \Gamma\left(\frac{1}{2}(a+m+1)\right) \Gamma\left(\frac{1}{2}(a-m+1)\right)} \\
& \times \frac{\Gamma\left(\frac{1}{2}(a-m+3)\right) \Gamma(a-m+1) \Gamma(b+1-m) \Gamma(c+1-m)}{\Gamma(a-b+1) \Gamma(a-c+1)} \\
& \left\{\frac{\Gamma(1+a-b) \Gamma(1+a-c)}{\Gamma(2-m+a) \Gamma(m+a-b-c)}-{ }_{4}^{(m-2)} F_{3}\left[\begin{array}{c}
a-m+1, b-m+1, \frac{1}{2}(a-m+3), c-m+1 \\
\frac{1}{2}(a-m+1), a-b+1, a-c+1
\end{array} ;-1\right]\right\}=\Omega_{9}
\end{aligned}
$$




$$
\begin{aligned}
& \text { (1.23) }
\end{aligned}
$$

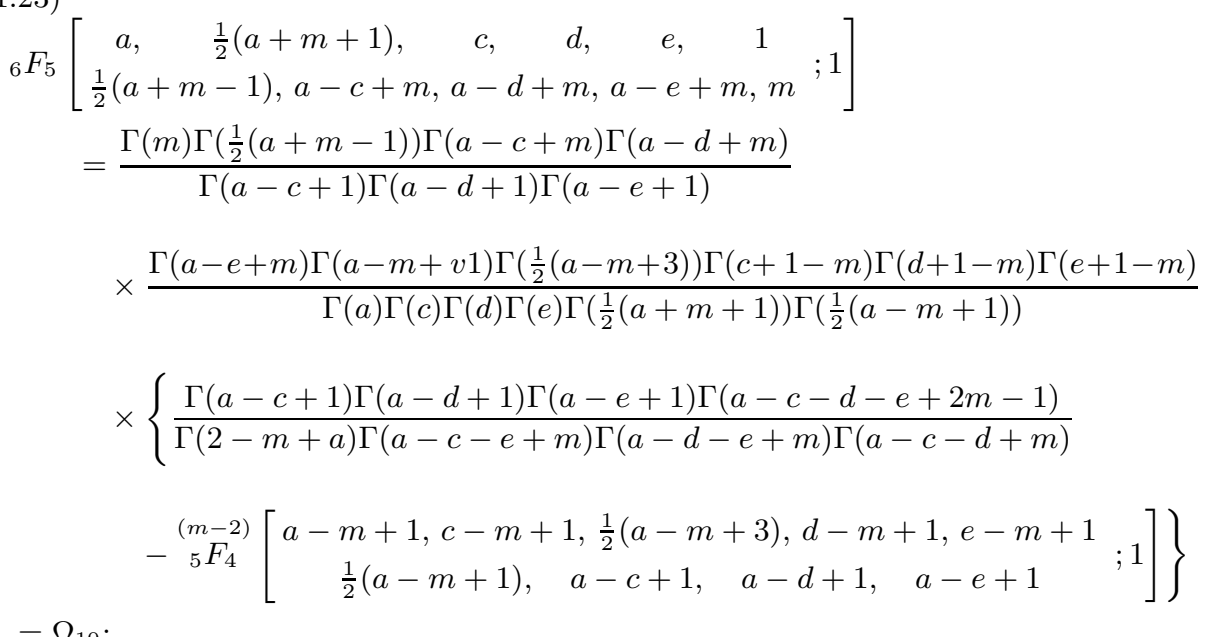$$
{ }_{8} F_{7}\left[\begin{array}{ccccc}
a, & \frac{1}{2}(a+m+1), \quad b, \quad c, \quad d, \quad 2 a-b-c-d+2 m-1+n, & m-n-1, & 1 \\
\frac{1}{2}(a+m-1), a-b+m, & a-c+m, a-d+m, b+c+d-a+1-m-n, a+n+1, m
\end{array}\right]
$$$$
=(-1)^{m-1}(m-1) ! \frac{\left(\frac{1}{2}(3-a-m)\right)_{m-1}(1-a+b-m)_{m-1}}{\left(\frac{1}{2}(1-a-m)\right)_{m-1}(1-a)_{m-1}}
$$$$
\times \frac{(1-a+c-m)_{m-1}(1-a+d-m)_{m-1}(m+n+a-b-c-d)_{m-1}(-a-n)_{m-1}}{(1-b)_{m-1}(1-c)_{m-1}(1-d)_{m-1}(b+c+d-2 a+2-2 m-n)_{m-1}(n+2-m)_{m-1}}
$$$$
\times\left\{\frac{(a-m+2)_{n}(a-b-c+m)_{n}(a-b-d+m)_{n}(a-c-d+m)_{n}}{(a-b+1)_{n}(a-c+1)_{n}(a-d+1)_{n}(a-b-c-d+2 m-1)_{n}}\right.
$$$$
\left.-{ }_{7} F_{6}\left[\begin{array}{c}
(m-2) \\
\frac{1}{2}\left(a-m+1, \frac{1}{2}(a-m+3), b-m+1, c-m+1, d-m+1,2 a-b-c-d+m+n,-n\right. \\
a-a-b+1, a-c+1, a-d+1, b+c+d-a+2-2 m-n, a-m+n+2
\end{array} ; 1\right]\right\}
$$$$
=\Omega_{11} \text {. }
$$

The aim of this paper is to establish eleven Eulerian's type integrals involving generalized hypergeomertic function by employing the summation formulas (1.14)-(1.24). Some known and several new and interesting special cases have also been given.

\section{Eulerian's type Integrals}

The eleven new class of Eulerian's type integrals involving generalized hypergeometric functions to be established in the paper are given in the following theorems. 
Theorem 2.1. For $m>1$ and $\operatorname{Re}(c-a-b+m)>1$, the following result

$$
\int_{0}^{1}(1-x)^{m-2} F_{1}\left[\begin{array}{cc}
a, b & b \\
c
\end{array} ; x\right] \mathrm{d} x=\frac{\Omega_{1}}{m-1}
$$

holds, where $\Omega_{1}$ is the same as given in (1.14).

Proof. For proving Theorem 2.1, denoting the left-hand side of (2.1) by $I$, expressing ${ }_{2} F_{1}$ as a series, change the order of integration and summation, evaluating the beta-integral, we have

$$
I=\sum_{n=0}^{\infty} \frac{(a)_{n}(b)_{n}}{(c)_{n} n !} \cdot \frac{\Gamma(1+n) \Gamma(m-1)}{\Gamma(m+n)} .
$$

Using (1.1) and after some simplification, we obtain

$$
I=\frac{\Gamma(m-1)}{\Gamma(m)} \sum_{n=0}^{\infty} \frac{(a)_{n}(b)_{n}(1)_{n}}{(c)_{n}(m)_{n}} \frac{1}{n !} .
$$

Summing up the series, we finally get

$$
I=\frac{1}{m-1}{ }_{3} F_{2}\left[\begin{array}{c}
a, b, 1 \\
c, m
\end{array} ; 1\right]
$$

Now, we observe that the ${ }_{3} F_{2}$ appearing can be evaluated with the help of the result (1.3) and we easily arrive at the right side of (2.1).

This completes the proof of Theorem 2.1.

Corollary 2.1. In Theorem 2.1, if we take $m=2,3$, we respectively get the following integrals.

$$
\int_{0}^{1}{ }_{2} F_{1}\left[\begin{array}{c}
a, b \\
c
\end{array} ; x\right] \mathrm{d} x=\frac{(c-1)}{(a-1)(b-1)}\left\{\frac{\Gamma(c-1) \Gamma(c-a-b+1)}{\Gamma(c-a) \Gamma(c-b)}-1\right\}
$$

provided $a, b \neq 1$ and $\operatorname{Re}(c-a-b)>-1$, and

$$
\begin{aligned}
\int_{0}^{1}(1-x)_{2} F_{1} & {\left[\begin{array}{c}
a, b \\
c
\end{array} ; x\right] \mathrm{d} x=\frac{(c-2)_{2}}{(a-2)_{2}(b-2)_{2}} } \\
& \times\left\{\frac{\Gamma(c-2) \Gamma(c-a-b+2)}{\Gamma(c-a) \Gamma(c-b)}-\frac{a b+c-2 a-2 b+2}{c-2}\right\},
\end{aligned}
$$

provided $\operatorname{Re}(c-a-b)>-2$.

Remark 2.1. These two results are recorded in the standard text of Exton [3, A.1.1.24 \& A.1.1.23, p. 155].

The following theorems and the corresponding corollaries 2.2 to 2.11 can be obtained by employing the results (1.4) to (1.13). Hence, they are given here without proof. 
Theorem 2.2. For $m>1$, the following result

$$
\int_{0}^{1}(1-x)^{m-2}{ }_{2} F_{1}\left[\begin{array}{c}
a, \quad b \\
a-b+m
\end{array} ;-x\right] \mathrm{d} x=\frac{\Omega_{2}}{m-1}
$$

holds, where $\Omega_{2}$ is the same as given in (1.15).

Corollary 2.2. In Theorem 2.2, if we take $m=2,3$, we respectively get the following integrals,

$$
\int_{0}^{1}{ }_{2} F_{1}\left[\begin{array}{c}
a, \quad b \\
a-b+2
\end{array} ;-x\right] \mathrm{d} x=\frac{(a-b+1)}{(a-1)(b-1)}\left\{1-\frac{\Gamma(1+a-b) \Gamma\left(\frac{1}{2} a+\frac{1}{2}\right)}{\Gamma(a) \Gamma\left(\frac{1}{2} a-b+\frac{3}{2}\right)}\right\}
$$

and

$$
\begin{aligned}
\int_{0}^{1}(1-x)_{2} F_{1}\left[\begin{array}{c}
a, \\
a-b+3
\end{array} ;-x\right] \mathrm{d} x \\
=\frac{(a-b+1)_{2}}{(a-2)_{2}(b-2)_{2}}\left\{\frac{\Gamma\left(\frac{1}{2} a\right) \Gamma(1+a-b)}{\Gamma(a-1) \Gamma\left(\frac{1}{2} a-b+2\right)}-\frac{3 a+b-a b-3}{1+a-b}\right\} .
\end{aligned}
$$

Theorem 2.3. For $m>1$, the following result

$$
\int_{0}^{1}(1-x)^{m-2}{ }_{2} F_{1}\left[\begin{array}{c}
a, \quad b \\
\frac{1}{2}(a+b+1)
\end{array} ; \frac{1}{2} x\right] \mathrm{d} x=\frac{\Omega_{3}}{m-1}
$$

holds, where $\Omega_{3}$ is the same as given in (1.16).

Corollary 2.3. If we take $m=2,3$ in Theorem 2.3, we get the following integrals:

$$
\int_{0}^{1}{ }_{2} F_{1}\left[\begin{array}{c}
a, \quad b \\
\frac{1}{2}(a+b+1)
\end{array} ; \frac{1}{2} x\right] \mathrm{d} x=\frac{(a+b-1)}{(a-1)(b-1)}\left\{\frac{\sqrt{\pi} \Gamma\left(\frac{1}{2}(a+b-1)\right)}{\Gamma\left(\frac{1}{2} a\right) \Gamma\left(\frac{1}{2} b\right)}-1\right\}
$$

and

$$
\begin{aligned}
& \int_{0}^{1}(1-x)_{2} F_{1}\left[\begin{array}{c}
a, b \\
\frac{1}{2}(a+b+1)
\end{array} ; \frac{1}{2} x\right] \mathrm{d} x \\
& =\frac{(a+b-1)(a+b-3)}{(a-2)_{2}(b-2)_{2}}\left\{\frac{\sqrt{\pi} \Gamma\left(\frac{1}{2}(a+b-3)\right)}{\Gamma\left(\frac{1}{2}(a-1)\right) \Gamma\left(\frac{1}{2}(b-1)\right)}-\frac{a b-a-b+1}{a+b-3}\right\},
\end{aligned}
$$

respectively.

Theorem 2.4. For $m>1$, the following result

$$
\int_{0}^{1}(1-x)^{m-2} F_{1}\left[\begin{array}{c}
a, 2 m-a-1 \\
b
\end{array} ; \frac{1}{2} x\right] \mathrm{d} x=\frac{\Omega_{4}}{m-1}
$$

holds, where $\Omega_{4}$ is the same as given in (1.17). 
Corollary 2.4. If we take $m=2,3$ in Theorem 2.4, we respectively get the following integrals:

$$
\int_{0}^{1}{ }_{2} F_{1}\left[\begin{array}{c}
a, 3-a \\
b
\end{array} ; \frac{1}{2} x\right] \mathrm{d} x=\frac{2(1-b)}{(1-a)_{2}}\left\{\frac{\Gamma\left(\frac{1}{2}(b-1)\right) \Gamma\left(\frac{1}{2} b\right)}{\Gamma\left(\frac{1}{2}(a+b)-1\right) \Gamma\left(\frac{1}{2}(b-a+1)\right)}-1\right\}
$$

and

$$
\begin{aligned}
\int_{0}^{1}( & -x)_{2} F_{1}\left[\begin{array}{c}
a, 3-a \\
b
\end{array} ; \frac{1}{2} x\right] \mathrm{d} x \\
\quad= & \frac{4(b-2)_{2}}{(a-4)_{4}}\left\{\frac{\Gamma\left(\frac{1}{2}(b-1)\right) \Gamma\left(\frac{1}{2}(b-2)\right)}{\Gamma\left(\frac{1}{2}(a+b)-2\right) \Gamma\left(\frac{1}{2}(b-a+1)\right)}-\frac{5 a-a^{2}+2 b-10}{2(b-2)}\right\} .
\end{aligned}
$$

Theorem 2.5. For $m>1$, the following result

$$
\int_{0}^{1}(1-x)^{m-2}{ }_{3} F_{2}\left[\begin{array}{c}
a, \quad b, \quad c \\
a-b+m, a-c+m
\end{array} ; x\right] d x=\frac{1}{m-1} \Omega_{5}
$$

holds, where $\Omega_{5}$ is the same as given in (1.18).

Corollary 2.5. If we take $m=2,3$ in Theorem 2.5, we respectively get the following integrals:

$$
\begin{aligned}
\int_{0}^{1}{ }_{3} F_{2}\left[\begin{array}{c}
a, \quad b, \quad c \\
a-b+2, a-c+2
\end{array} ; x\right] \mathrm{d} x=\frac{(1+a-b)(1+a-c)}{(a-1)(b-1)(c-1)} \\
\times\left\{\frac{\Gamma\left(\frac{1}{2}(a+1)\right) \Gamma(1+a-b) \Gamma(1+a-c) \Gamma\left(\frac{1}{2} a-b-c+\frac{5}{2}\right)}{\Gamma(a) \Gamma\left(\frac{1}{2} a-b+\frac{3}{2}\right) \Gamma\left(\frac{1}{2} a-c+\frac{3}{2}\right) \Gamma(2+a-b-c)}-1\right\}
\end{aligned}
$$

and

$$
\begin{gathered}
\int_{0}^{1}(1-x)_{3} F_{2}\left[\begin{array}{c}
a, \quad b, \quad c \\
a-b+3, a-c+3
\end{array} ; x\right] \mathrm{d} x=\frac{(a-b+1)_{2}(a-c+1)_{2}}{(a-2)_{2}(b-2)_{2}(c-2)_{2}} \\
\times\left\{\frac{\Gamma\left(\frac{1}{2} a\right) \Gamma(1+a-b) \Gamma(1+a-c) \Gamma\left(\frac{1}{2} a-b-c+4\right)}{\Gamma(a-1) \Gamma\left(\frac{1}{2} a-b+2\right) \Gamma\left(\frac{1}{2} a-c+2\right) \Gamma(3+a-b-c)}\right. \\
\left.-\frac{(a-2)(b-2)(c-2)}{(a-b+1)(a-c+1)}-1\right\} .
\end{gathered}
$$

Theorem 2.6. For $m>1$ and $\operatorname{Re}(2 c-a-b)>-1$, the following result

$$
\int_{0}^{1}(1-x)_{3}^{m-2} F_{2}\left[\begin{array}{c}
a, \quad b, \quad c \\
\frac{1}{2}(a+b+1), 2 c+1-m
\end{array} ; x\right] \mathrm{d} x=\frac{\Omega_{6}}{m-1}
$$

holds, where $\Omega_{6}$ is the same as given in (1.19).

Corollary 2.6. If we take $m=2,3$ in Theorem 2.6, we respectively obtain the following integrals:

$$
\begin{aligned}
& \int_{0}^{1}{ }_{3} F_{2}\left[\begin{array}{cl}
a, \quad b, & c \\
\frac{1}{2}(a+b+1), & 2 c-1
\end{array} ; x\right] \mathrm{d} x \\
& =\frac{(a+b-1)}{(a-1)(b-1)}\left\{\frac{\sqrt{\pi} \Gamma\left(c-\frac{1}{2}\right) \Gamma\left(\frac{1}{2}(a+b-1)\right) \Gamma\left(c-\frac{1}{2}(a+b-1)\right)}{\Gamma\left(\frac{1}{2} a\right) \Gamma\left(\frac{1}{2} b\right) \Gamma\left(c-\frac{1}{2} a\right) \Gamma\left(c-\frac{1}{2} b\right)}-1\right\}
\end{aligned}
$$


and

$$
\begin{aligned}
& \int_{0}^{1}(1-x)_{3} F_{2}\left[\begin{array}{cl}
a, \quad b, & c \\
\frac{1}{2}(a+b+1), & 2 c-2
\end{array} ; x\right] \mathrm{d} x=\frac{(2 c-3)(a+b-1)(a+b-3)}{(c-1)(a-2)_{2}(b-2)_{2}} \\
& \times\left\{\frac{\sqrt{\pi} \Gamma\left(c-\frac{3}{2}\right) \Gamma\left(\frac{1}{2}(a+b-3)\right) \Gamma\left(c-\frac{1}{2}(a+b-1)\right)}{\Gamma\left(\frac{1}{2}(a-1)\right) \Gamma\left(\frac{1}{2}(b-1)\right) \Gamma\left(c-\frac{1}{2}(a+1)\right) \Gamma\left(c-\frac{1}{2}(b+1)\right)}-\frac{(a-2)(b-2)}{a+b-3}-1\right\} .
\end{aligned}
$$

Theorem 2.7. For $m>1$, the following result

$$
\int_{0}^{1}(1-x)_{3}^{m-2} F_{2}\left[\begin{array}{cc}
a, 2 m-1-a, b \\
c, 2 b-c+1
\end{array} ; x\right] \mathrm{d} x=\frac{\Omega_{7}}{m-1},
$$

holds, where $\Omega_{7}$ is the same as given in (1.20).

Corollary 2.7. If we take $m=2,3$ in Theorem 2.7, we respectively get the following integrals:

$$
\begin{aligned}
& \int_{0}^{1}{ }_{3} F_{2}\left[\begin{array}{c}
a, 3-a, b \\
c, 2 b-c+1
\end{array} ; x\right] \mathrm{d} x=\frac{(c-1)(c-2 b)}{(a-2)_{2}(b-1)} \\
& \quad \times\left\{\frac{\pi 2^{3-2 b} \Gamma(c-1) \Gamma(2 b-c)}{\Gamma\left(\frac{1}{2}(a+c)-1\right) \Gamma\left(b+\frac{1}{2}(a-c-1)\right) \Gamma\left(\frac{1}{2}(1-a+c)\right) \Gamma\left(b+1-\frac{1}{2}(a+c)\right)}-1\right\}
\end{aligned}
$$

and

$$
\begin{gathered}
\int_{0}^{1}(1-x){ }_{3} F_{2}\left[\begin{array}{c}
a, 3-a, b \\
c, 2 b-c+1
\end{array} ; x\right] \mathrm{d} x \\
=\left\{\frac{\pi 2^{4-2 b} \Gamma(c-2) \Gamma(2 b-c+1)}{\Gamma\left(\frac{1}{2}(a+c)-2\right) \Gamma\left(b+\frac{1}{2}(a-c-3)\right) \Gamma\left(\frac{1}{2}(1-a+c)\right) \Gamma\left(b+1-\frac{1}{2}(a+c)\right)}\right. \\
\left.-\frac{(a-2)(3-a)(b-2)}{2(c-2)(2 b-c-1)}-\frac{1}{2}\right\} .
\end{gathered}
$$

Theorem 2.8. For $m>1$, the following result

$$
\int_{0}^{1}(1-x)^{m-2}{ }_{3} F_{2}\left[\begin{array}{c}
a, b,-n+m-1 \\
c, 1+a+b-c-n
\end{array} ; x\right] \mathrm{d} x=\frac{\Omega_{8}}{m-1},
$$

holds. where $\Omega_{8}$ is the same as given in (1.21).

Corollary 2.8. In Theorem 2.8, if we take $m=2,3$, we respectively get the following integrals.

$\int_{0}^{1}{ }_{3} F_{2}\left[\begin{array}{c}a, b,-n+1 \\ c, 1+a+b-c-n\end{array} ; x\right] \mathrm{d} x=\frac{(1-c)(c-a-b+n)}{n(1-a)(1-b)}\left\{\frac{(c-a)_{n}(c-b)_{n}}{(c)_{n}(c-a-b+1)_{n}}-1\right\}$

and

$$
\begin{aligned}
\int_{0}^{1}(1-x)_{3} F_{2} & {\left[\begin{array}{c}
a, b,-n+2 \\
c, 1+a+b-c-n
\end{array} ; x\right] \mathrm{d} x=\frac{(1-c)_{2}(c-a-b+n)_{2}}{n(1-a)_{2}(1-b)_{2}} } \\
\times & \left\{\frac{(c-a)_{n}(c-b)_{n}}{(c-2)_{n}(c-a-b+2)_{n}}+\frac{n(a-2)(b-2)}{(c-2)(a+b-c-n-1)}-1\right\} .
\end{aligned}
$$


Theorem 2.9. For $m>1$ the following result

$$
\begin{aligned}
& \int_{0}^{1}(1-x)^{m-2}{ }_{4} F_{3}\left[\begin{array}{c}
a, b, \\
\frac{1}{2}(a+m-1), a-b+m, a-c+m
\end{array} ;-x\right] \mathrm{d} x \\
& =\frac{\Omega_{9}}{m-1}
\end{aligned}
$$

holds, where $\Omega_{9}$ is the same as given in (1.22).

Corollary 2.9. If we take $m=2,3$ in Theorem 2.9, we respectively get the following integrals:

$$
\begin{aligned}
\int_{0}^{1}{ }_{4} F_{3}\left[\begin{array}{c}
a, \quad \frac{1}{2}(a+3), b, b \\
\frac{1}{2}(a+1), \\
a-b+2, a-c+2
\end{array} ;-x\right] \mathrm{d} x \\
=\frac{4(1+a-b)(1+a-c)}{\left(a^{2}-1\right)(a-1)(b-1)(c-1)}\left\{1-\frac{\Gamma(1+a-b) \Gamma(1+a-c)}{\Gamma(a) \Gamma(2+a-b-c)}\right\}
\end{aligned}
$$

and

$$
\begin{aligned}
& \int_{0}^{1}(1-x){ }_{4} F_{3}\left[\begin{array}{c}
a, \frac{1}{2}(a+4), b, c \\
\frac{1}{2}(a+3), a-b+3, a-c+3
\end{array} ;-x\right] \mathrm{d} x \\
& =\frac{(1+a-b)_{2}(1+a-c)_{2}}{(a+2)(b-2)_{2}(c-2)_{2}}\left\{\frac{\Gamma(1+a-b) \Gamma(1+a-c)}{\Gamma(a) \Gamma(3+a-b-c)}+\frac{(b-1)(c-1)-a}{(a-b+1)(a-c+1)}\right\} .
\end{aligned}
$$

Theorem 2.10. For $m>1$, the following result

$$
\begin{aligned}
& \int_{0}^{1}(1-x)^{m-2}{ }_{5} F_{4}\left[\begin{array}{c}
a, \quad \frac{1}{2}(a+m+1), c, d, e \\
\frac{1}{2}(a+m-1), a-c+m, a-d+m, a-e+m
\end{array} ; x\right] \mathrm{d} x \\
& \quad=\frac{\Omega_{10}}{m-1}
\end{aligned}
$$

holds, where $\Omega_{10}$ is the same as given in (1.23).

Corollary 2.10. If we take $m=2,3$ in Theorem 2.10, we respectively obtain the following integrals:

$$
\begin{aligned}
\int_{0}^{1}{ }_{5} F_{4} & {\left[\begin{array}{c}
a, \quad \frac{1}{2}(a+3), \quad c, \quad d, \quad e \\
\frac{1}{2}(a+1), a-c+2 a-d+2, a-e+2
\end{array} ; x\right] \mathrm{d} x } \\
= & \frac{(1+a-c)(1+a-d)(1+a-e)}{(1+a)(c-1)(d-1)(e-1)} \\
& \times\left\{\frac{\Gamma(1+a-c) \Gamma(1+a-d) \Gamma(1+a-e) \Gamma(3+a-c-d-e)}{\Gamma(a) \Gamma(2+a-d-e) \Gamma(2+a-c-e) \Gamma(2+a-c-d)}-1\right\}
\end{aligned}
$$


and

$$
\begin{aligned}
& \int_{0}^{1}(1-x){ }_{5} F_{4}\left[\begin{array}{ccc}
a, & \frac{1}{2}(a+4), \quad c, \quad d, \quad e \\
\frac{1}{2}(a+2), & a-c+3, a-d+3, & a-e+3
\end{array} ; x\right] \mathrm{d} x \\
& =\frac{(1+a-c)_{2}(1+a-d)_{2}(1+a-e)_{2}}{(a-1)(a+2)(c-2)_{2}(d-2)_{2}(e-2)_{2}} \\
& \times\left\{\frac{\Gamma(1+a-c) \Gamma(1+a-d) \Gamma(1+a-e) \Gamma(5+a-c-d-e)}{\Gamma(a-1) \Gamma(3+a-d-e) \Gamma(3+a-c-e) \Gamma(3+a-c-d)}\right. \\
& \left.-\frac{a(c-2)(d-2)(e-2)}{(1+a-c)(1+a-d)(1+a-e)}\right\} \text {. }
\end{aligned}
$$

Theorem 2.11. Let

$$
\begin{aligned}
& \left(a_{1}, a_{2}, a_{3}, a_{4}, a_{5}, a_{6}, a_{7}\right)=\left(a, \frac{1}{2}(a+m+1), b, c, d,\right. \\
& 2 a-b-c-d+2 m+n-1, m-n-1), \\
& \left(b_{1}, b_{2}, b_{3}, b_{4}, b_{5}, b_{6}\right)=\left(\frac{1}{2}(a+m-1), a-b+m, a-c+m, a-d+m,\right. \\
& b+c+d-a-m-n+1, a+n+1) .
\end{aligned}
$$

Then, for $m>1$ we have the following result

$$
\int_{0}^{1}(1-x)^{m-2}{ }_{7} F_{6}\left[\begin{array}{c}
a_{1}, a_{2}, a_{3}, a_{4}, a_{5}, a_{6}, a_{7} \\
b_{1}, b_{2}, b_{3}, b_{4}, b_{5}, b_{6}
\end{array} ; x\right] \mathrm{d} x=\frac{\Omega_{11}}{m-1},
$$

where $\Omega_{11}$ is the same as given in (1.24).

Corollary 2.11. If we take $m=2,3$ in Theorem 2.11, we respectively get the following integrals:

$$
\begin{gathered}
\int_{0}^{1}{ }_{7} F_{6}\left[\begin{array}{c}
a, \frac{1}{2}(a+3), b, c, d, 2 a-b-c-d+n+3,1-n, \\
\frac{1}{2}(a+1), 2+a-b, 2+a-c, 2+a-d, b+c+d-a-n-1, a+n+1
\end{array} ; x\right] \mathrm{d} x \\
=\frac{(b-a-1)(c-a-1)(d-a-1)(n+2+a-b-c-d)(a+n)}{n(1+a)(1-b)(1-c)(1-d)(b+c+d-2 a-2-n)} \\
\times\left\{1-\frac{(a)_{n}(a-b-c+2)_{n}(a-b-d+2)_{n}(a-c-d+2)_{n}}{(1+a-b)_{n}(1+a-c)_{n}(1+a-d)_{n}(3+a-b-c-d)_{n}}\right\}
\end{gathered}
$$

and

$$
\begin{aligned}
\int_{0}^{1}(1-x)_{7} F_{6}\left[\begin{array}{c}
a, \frac{1}{2}(a+4), b, c, d, 2 a-b-c-d+n+5,2-n \\
\frac{1}{2}(a+2), 3+a-b, 3+a-c, 3+a-d, b_{5}, a+n+1
\end{array} ; x\right] \mathrm{d} x \\
=\frac{(a-2)(b-a-2)_{2}(c-a-2)_{2}(d-a-2)_{2}(-a-n)_{n}(3+n+a-b-c-d)_{2}}{2(a+2)(1-a)_{2}(1-b)_{2}(1-c)_{2}(1-d)_{2}(n-1)_{2}(b+c+d-2 a-4-n)_{2}} \\
\quad \times\left\{\frac{(a-1)_{n}(a-b-c+3)_{n}(a-b-d+3)_{n}(a-c-d+3)_{n}}{(a-b+1)_{n}(a-c+1)_{n}(a-d+1)_{n}(a-b-c-d+5)_{n}}\right. \\
\left.+\frac{n a(b-2)(c-2)(d-2)(2 a-b-c+n+3)}{(a-b+1)(a-c+1)(a-d+1)(b+c+d-a-n-4)(n+a-1)}-1\right\},
\end{aligned}
$$

where $b_{5}=b+c+d-a-n-2$. 
Similarly other results can be obtained.

\section{Conclusion Remark}

In this paper, eleven Eulerian's type integrals involving generalized hypergeometric functions have been evaluated in terms of gamma function by employing very recently obtained summation theorems by Masjed-Jamei and Koepf. Several new, interesting and elementary integrals have also been given as special cases of our main findings.

\section{REF E R E N C ES}

1. G. E. Andrews, R. Askey, R. Roy: Special Functions. Cambridge University Press, Cambridge, 1999.

2. W. N. BAILEY: Generalized Hypergeometric Series. Cambridge University Press, Cambridge, 1935; Reprinted by Stechert-Hafner, New York, 1964.

3. H. Exton: Handbook of Hypergeometric Integrals. Ellis Horwood Publishers, Chichester, 1978.

4. M. Masjed-JAmei, W. KoepF: Some summation theorems for generalized hypergeometric functions. Axioms 2018, 7(2), 38; https://doi.org/ 10.3390/axioms7020038.

5. A. M. Mathai, R. K. Saxena: Generalized Hypergeometric Functions with Applications in Statistics and Physical Sciences. Lecture Note in Mathematics, Springer: Berlin/Heidelberg, Germany; New York, NY, USA, 1973; Volume 348.

6. A. F. Nikiforov, V. B. Uvarov: Special Functions of Mathematical Physics. A Unified Introduction with Applications. Birkhäuser: Basel, Switzerland, 1988.

7. A. P. Prudnikov, Yu. A. Brychkov, O. I. Marichev: More Special Functions: Integrals and Series. vol. 3, Gordon and Breach Science Publishers, Amsterdam, The Netherlands, 1990.

8. E. D. Rainville: Special Functions. The Macmillan Company, New York, 1960; Reprinted by Chelsea Publishing Company, Bronx, New York, 1971.

9. L. J. Slater: Generalized Hypergeometric Functions. Cambridge University Press, Cambridge, UK, 1966.

Insuk Kim

Department of Mathematics Education, Wonkwang University

Iksan, 570-749, Republic of Korea

iki@wku.ac.kr

Gradimir V. Milovanović

Serbian Academy of Sciences and Arts, 11000 Beograd, Serbia

Faculty of Sciences and Mathematics, University of Niš

18000 Nis, Serbia

gvm@mi.sanu.ac.rs 
Arjun K. Rathie

Department of Mathematics

Vedant College of Engineering and Technolology

(Rajasthan Technical University)

Bundi, 323021, Rajasthan, India

arjunkumarrathie@gmail.com 